\title{
Influence of Shield Construction on Close-Access Pile Group Foundation of Bridge
}

\author{
Feng Xingren ${ }^{1}$, She Mingkang ${ }^{2}$,Chen Baoguo*2 ${ }^{\star 2}$ Mao Xinying ${ }^{2}$,Jiang
}

Chengxuan $^{2}$

(1. Sinohydro Bureau 7 Co, Ltd, Chengdu 610081, China ;2. Engineering Faculty, China University of Geosciences, Wuhan 430074, China)

Keywords: Shield Construction; Close-Access; Pile Group Foundation; Numerical Simulation

Abstract: The influence of shield tunneling on close-access existing pile group foundation of bridge has always been a difficult engineering problem. To analyze this problem, the finite difference software FLAC3D was conducted to simulate the whole process of shield crossing the adjacent pile group foundation of bridge. The distribution and variation law of ground surface settlement, as well as the deformation law and stress characteristics of pile group foundation were discussed. The research results show that: (1) The settlement of ground surface decrease with the distance close to the edge of the cap of pile group foundation due to the shielding effect of pile group foundation and constraint to soil layer; (2) The horizontal displacement of each foundation pile gradually increases, then tends to be stable during the shield crossing process, the horizontal displacement increment reach to a peak value when the shield construction in the range of $6 \mathrm{~m}$ before and after the foundation pile; (3) The maximum axial force in foundation pile decrease with the distance between the foundation pile and shield tunnel. The distribution of axial force in foundation pile increase with the pile depth and then decrease due to the negative skin friction induce by shield construction. (4) There are three reverse bending points in each foundation pile due to the influence of shield construction, the bending moment in foundation pile decrease with the spacing between the foundation pile and shield tunnel.

\section{Introduction}

In the process of urban rail transit construction, the subway tunnel will inevitably pass through the near bridge pile group foundation. The shield construction may cause the stratum soil loss, the water pore pressure variation, as well as the tunnel lining deformation, these will resulting in deformation of the stratum in different degrees, which induces the change of the soil stress state around the pile group foundation of bridge and causes the negative skin friction on the foundation pile. This will weaken the bearing capacity of the pile foundation and even threatens the stability of the bridge.

Some researches have been carried out relevant to the influence of shield tunneling on near pile foundation. J.D.Morton et al. ${ }^{[1]}$ use the laboratory model experiment to study the influence of tunnel construction on the pile foundation, which has some guiding significance to practical project. N.Loganathan ${ }^{[2]}$ through the centrifuge test found that tunnel excavation will change the axial force and bending moment of adjacent piles, and points out its influence mainly depends on the distance of pile and tunnel and the relationship of pile length and tunnel depth. Ong et al. ${ }^{[3]}$ use centrifuge model test method to study the influence of tunnel excavation on adjacent single pile and tunnel deformation at the arch line. Lee et al. ${ }^{[4]}$ applied three dimensional elastoplastic finite element technique to investigate the interaction of tunnel pile and the influence of tunnel excavation on adjacent piles. Fang Yong ${ }^{[5]}$ considered the dynamic tunneling of shield in numerical simulation, 
and studied the influence of Shield tunneling parameters on the displacement of adjacent pile. Luo Wenlin et al. ${ }^{[6]}$ analyzed the influence of tunnel excavation on pile foundation by studying the parameters of the FLAC3D pile element structure. Zhao Zhifeng et al. ${ }^{[7 \sim 9]}$ analyzed the deformation and internal force of the bridge foundation pile by numerical simulation of shield tunneling.

A shield tunnel exactly crossing an bridge pile group foundation which construction in the reclamation silt stratum In Shenzhen City of China. Because of the weakness of the ground soil and the interactive between pile group foundation and shield construction, the stability of the bridge and shield tunnel is very important. Therefore, a numerical simulation was conducted to investigate the performance of the shield tunnel crossing the pile group foundation of the bridge based on the practical engineering.

\section{Numerical simulation}

\section{Computational model}

The problem selected for the numerical analysis is related to the exiting of an bridge pile group foundation as a part of Shenzhen Metro Line 5. In the the area of the problem, the diameter of the tunnel is $6 \mathrm{~m}$, and the distance between the tunnel and the ground is $16.61 \mathrm{~m}$. The bridge pile cap is $42.6 \mathrm{~m}$ in length, $18 \mathrm{~m}$ in width and $5 \mathrm{~m}$ in height. The pile foundation adopts the bored pile with a diameter of $1.9 \mathrm{~m}$, which is $33.4 \mathrm{~m}$, and the minimum net distanced between the pile and the tunnel is $1.5 \mathrm{~m}$. Tunnel lining segment is made of reinforced concrete material with an inner diameter of $5.4 \mathrm{~m}$, a thickness of $0.3 \mathrm{~m}$ and a ring width of $1.5 \mathrm{~m}$. In the course of shield tunneling, the shield tail space, $0.1 \mathrm{~m}$ thick, between the segment and the soil is grouted with cement paste. The strata are mainly divided into four layers, from the ground surface down to plain fill (rubble), silt, hard plastic sandy clayey soil and all weathered gneiss mixed granite. The relation between the shield tunnel and the adjacent pile foundation is shown in Figure 1 and Fig. 2.

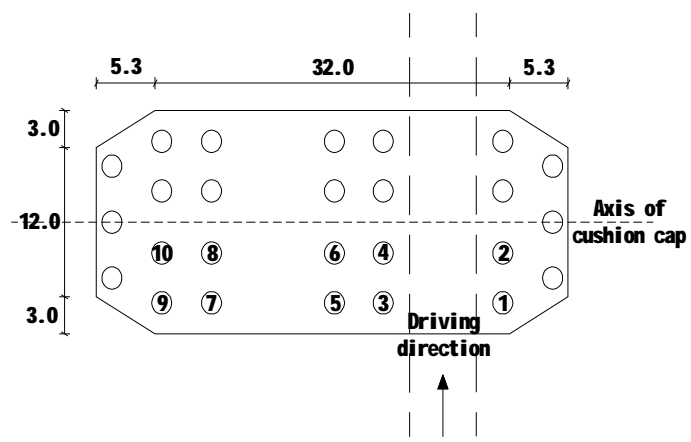

Fig. 1 plane diagram of relation between shield tunnel and pile foundation (unit: $\mathrm{m}$ )

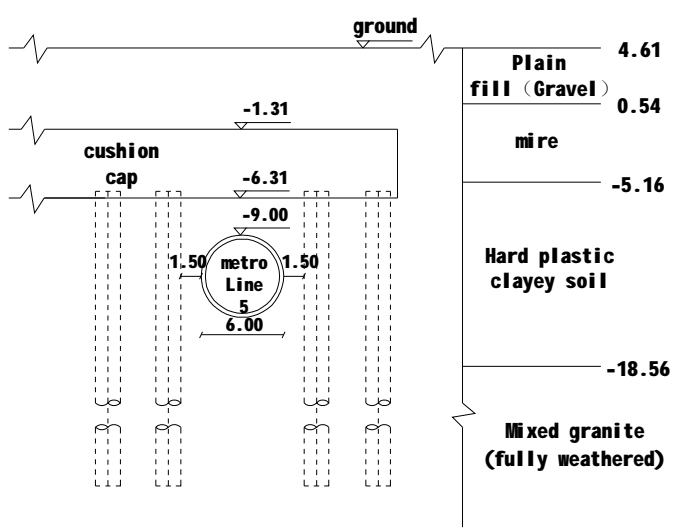

Fig. 2 schematic diagram of the relationship between Shield tunneling and pile foundation (unit: $\mathrm{m}$ ) 


\section{Numerical modeling and boundary conditions}

According to the engineering example and research ${ }^{[10]}$, it is found that shield tunneling has little influence on the soil mass outside the 3D 5D (D represents tunnel diameter) of the tunnel center, which can be neglected. The calculation model is $90 \mathrm{~m}$ in length, $48 \mathrm{~m}$ in width and $45 \mathrm{~m}$ in height, totaling 55992 entity units and 59816 nodes. In the model, the soil, the pile cap and the cement paste are all made of entity unit, the segment is made of shell structural unit, the cast-in-place pile is made of pile structural unit, and the material parameters are shown in table 1. The soil layer is represented by an elastic-plastic model with the Mohr-Coulomb failure criterion, while the pile cap, the pile and the segment is assumed to be linearly elastic materials.

In the numerical model, the two side boundaries of the model were horizontally restrained, while the horizontal and vertical displacements of the model bottom were fixed. The load on the bridge pile foundation includes the weight of bridge body, the load of pavement and so on, which can be simplified to the vertical uniform load on the top surface of the pile cap, and its value is $50 \mathrm{kPa}$.

Table 1. Material parameters of the model

\begin{tabular}{cccccc}
\hline Materials & $\begin{array}{c}\text { Bulk } \\
\text { density/ } \\
\left(\mathrm{kN} / \mathrm{m}^{3}\right)\end{array}$ & $\begin{array}{c}\text { Cohesion/ } \\
(\mathrm{kPa})\end{array}$ & $\begin{array}{c}\text { Friction/ } \\
(\mathrm{o})\end{array}$ & $\begin{array}{c}\text { Poisson's } \\
\text { ratio }\end{array}$ & $\begin{array}{c}\text { Modulus of } \\
\text { elasticity/ } \\
(\mathrm{MPa})\end{array}$ \\
\hline Soil filling (crushed stone) & 19.2 & 0 & 35 & 0.24 & 7.5 \\
\hline Silt & 15.6 & 10 & 3.5 & 0.42 & 1.8 \\
\hline $\begin{array}{c}\text { Hard plastic sand clayey } \\
\text { soil }\end{array}$ & 18.4 & 20 & 20.5 & 0.28 & 28 \\
\hline $\begin{array}{c}\text { All weathered slices of } \\
\text { mixed granite }\end{array}$ & 21.3 & 32 & 24.5 & 0.28 & 70 \\
\hline Segment & 25 & $/$ & $/$ & 0.2 & 30000 \\
\hline Cement paste & 20.5 & $/$ & $/$ & 0.3 & 15 \\
\hline Pile & 25 & $/$ & $/$ & 0.2 & 28000 \\
\hline Pile caps & 25 & $/$ & $/$ & 0.2 & 30000 \\
\hline
\end{tabular}

\section{Numerical simulation process}

The simulation process is divided into three steps: (1) After setting the artificial boundary and the material attribute assignment, the initial geostress is achieved by assigning gravity to all the soil units; (2) After the displacement field and velocity field are cleared, the bridge pile foundation is simulated by the structural unit of the software, change the soil unit parameters to simulate the pile cap, and set the concentrated load on the top surface of the pile cap, calculating the soil stress state under gravity; (3) The tunnel shield is excavated, after the displacement field and velocity field are cleared.

In the process of Shield tunneling, the jacking force exerted on the palm surface is $0.13 \mathrm{MPa}$, and by killing the grouting layer unit to simulate the shield tail void. While killing the grouting unit, a grouting pressure of $0.15 \mathrm{MPa}$ is applied in the radial direction, and the grouting layer and the segment lining (shell) unit are activated in the next calculation step. The shield tunneling forward $48 \mathrm{~m}$, which has 16 steps and $3 \mathrm{~m}$ for each step. Among them, the step $1 \sim$ step 5 belongs to the process of the shield approaching pile group foundation, the step $6 \sim$ step11 belongs to the process of shield passing through group piles, and the step12 step16 belongs to the process of the shield away from the pile foundation. 


\section{Calculation Results and Analysis}

\section{Analysis of surface subsidence}

In the process of shield tunneling (gradually approaching the pile cap), the distribution and variation law of ground surface settlement as shown in Figure 3. It is shown that the top of tunnel is the maximum ground surface settlement, on both sides of the settlement gradually decay, forming a transverse settlement trough, showing the approximate Gauss distribution, and Peck ${ }^{[11]}$ the theoretical results shows that the numerical simulation method is reasonable and feasible.

In the process of gradually approaching the pile cap of the pile foundation (Fig. 3), the ground subsidence gradually decreases; the distance from $21 \mathrm{~m}$ to $3 \mathrm{~m}$, the surface subsidence above the tunnel is reduced from about $7.5 \mathrm{~mm}$ to about $0.5 \mathrm{~mm}$. This is due to the shielding effect of the pile caps and pile groups, reducing the disturbance of shield construction to nearby soil. Therefore, the pile group foundation of the bridge plays a strengthening role in the surrounding soil and restrains the deformation of the surrounding soil.

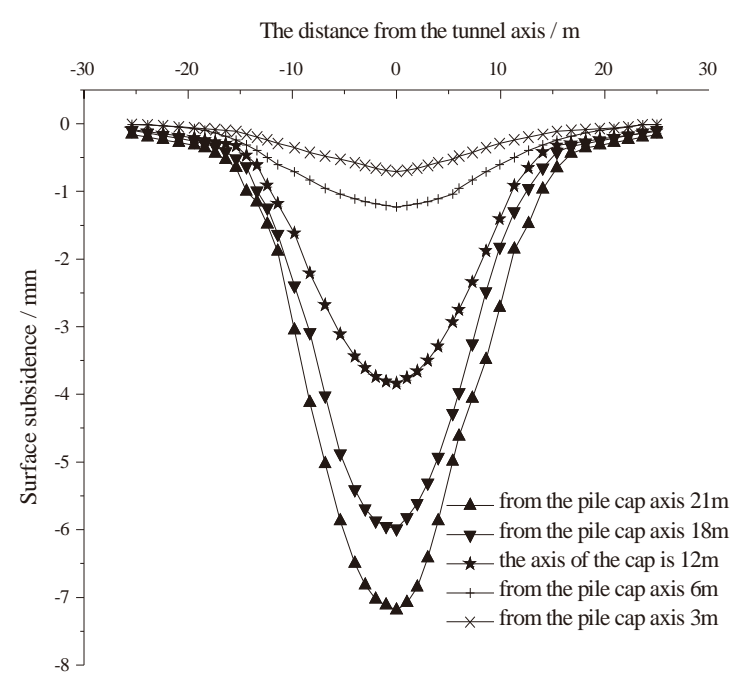

Fig. 3 The law of transverse surface subsidence deformation

\section{Analysis of group Pile deformation}

Shield construction also has some influence on the deformation of pile foundation. According to numerical simulation, the maximum settlement of pile top caused by tunnel shield excavation is $0.44 \mathrm{~mm}$. Because the pile foundation of the bridge is an end bearing pile, and the pile end reaches the mixed granite stratum, the influence of shield construction on the settlement of the bridge pile foundation is limited. The settlement of the pile head may be caused by the increase of the frictional resistance that is due to the local lack of soil during the construction. In addition, by studying the 10 piles as shown in Figure 1 (1\#, 3\#, 5\#, 7\# and 9\# pile belong to side piles; 2\#, 4\#, 6\#, 8\# and 10 pile belong to the row piles), it is found that there is shelter effect in the pile group foundation.

The distribution and variation regularity of horizontal displacements of $1 \# \sim 10 \#$ piles in shield tunneling are shown in Figure 4. The horizontal displacements of the 1\#, 3\#, 5\# and 7\# piles are higher than those of the $2 \#, 4 \#, 6 \#, 8 \#$ and $10 \#$ piles in the same column., which indicates that the shelter effect in the pile group foundation has a great influence on the horizontal displacement. 


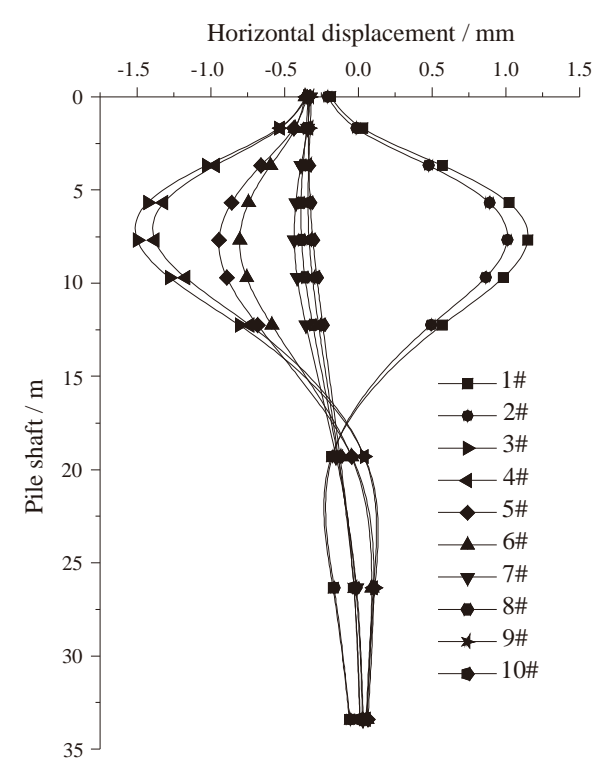

Fig. 4 Horizontal position of each base pile

The horizontal displacement of the shield passing through the 1\# pile as shown in Figure 5. It is can be seen from the figure that at step2, there is almost no horizontal displacement of the pile; From step2 to step4, the maximum horizontal displacement of pile increases by $0.093 \mathrm{~mm}$; From step4 to step6, the maximum horizontal displacement of the pile shaft increases $0.465 \mathrm{~mm}$; From step6 to step8, the maximum horizontal displacement of the pile shaft increases $0.582 \mathrm{~mm}$; From step8 to step10, the maximum horizontal displacement of the pile shaft increases $0.011 \mathrm{~mm}$; After step 10, the horizontal displacement of the pile is basically stable. Therefore, the influence of shield tunneling on the horizontal displacement of pile mainly occurs within the range of $6 \mathrm{~m}$ before and after the pile.

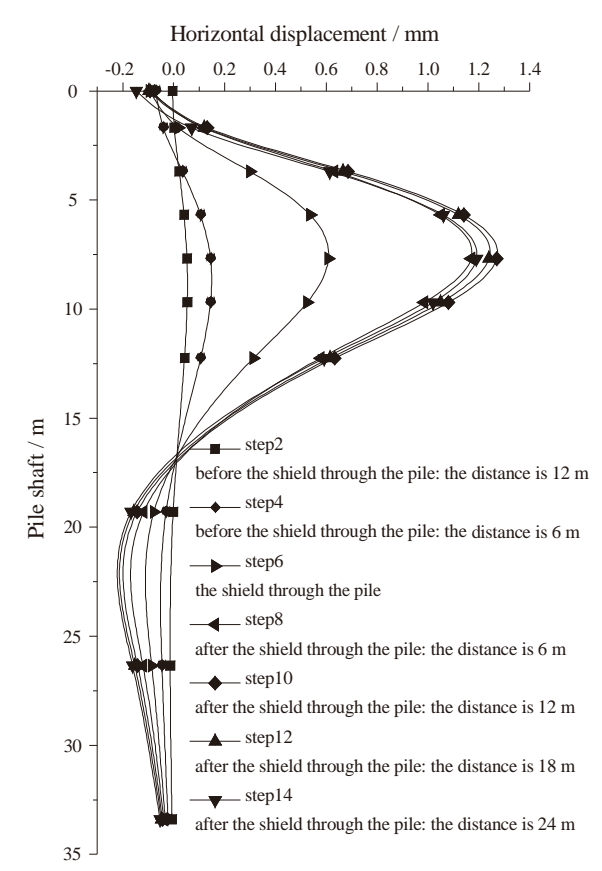

Fig. 5 Horizontal displacement of $1 \#$ piles in the whole process of shield tunneling

The distribution and variation regularity of axial force of the shield passing through the $1 \#$ $10 \#$ pile foundation as shown in Figure 6. It can be seen from the diagram that the axial force of the $1 \#, 3 \#, 5 \#, 7 \#$ and $9 \#$ piles is larger than that of the $2 \#, 4 \#, 6 \#, 8 \#$ and 10\# piles, which indicates that 
the shadowing effect in the group pile foundation has a great influence on the axial force. Comparing the net spacing between piles(3\#, 5\#, 7\# and 9\# pile) and tunnel, the net spacing increases gradually with the increase of stake number, and the corresponding pile axial force decreases, indicating that the net spacing between the foundation pile and the tunnel is larger, the corresponding pile axial force is smaller.
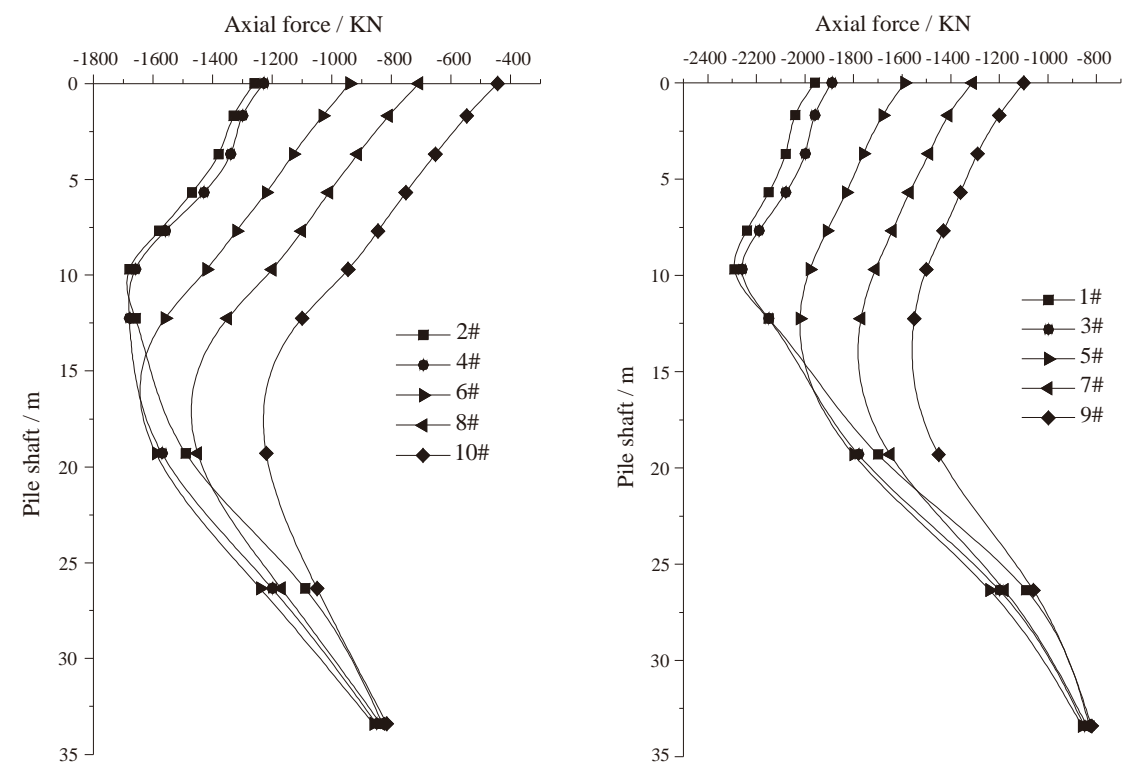

Fig. 6 Distribution of the Axial Force Fx along the pile shaft of each base pile

The axial force variation trend of each pile is basically the same, and it increases first and then decreases along the pile depth. This is mainly affected by the shield construction, the upper part of the base pile produces the negative skin friction, which leads to the increase of axial force. And the lower part of the base pile produces the positive skin friction, which leads to the reduction of axial force. In addition, as the disturbing degree of each pile is different, the maximum axial force position is also different.

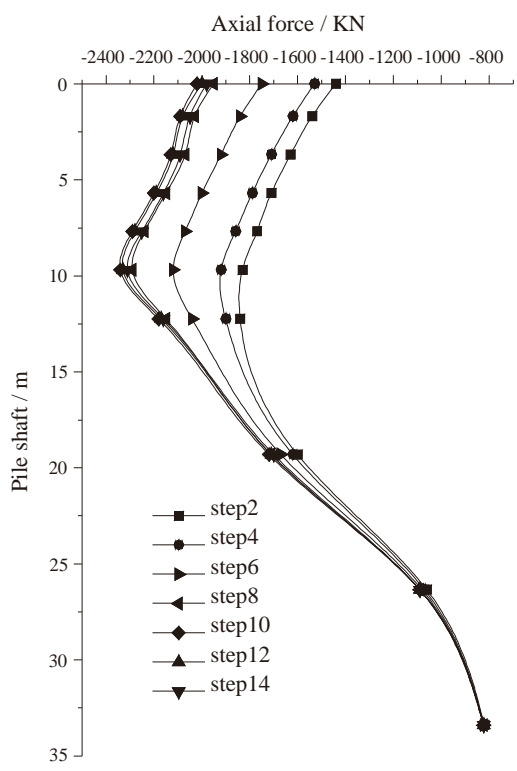

Fig. 7 Axial force of 1\# pile in the whole process of shield tunneling

The axial force variation curve of the shield passing through the 1\# pile as shown in Figure 7. It can be seen from the figure that from step2 to step4, the maximum axial force increases $50 \mathrm{KN}$; 
From step4 to step6, the maximum axial force increases $100 \mathrm{KN}$; From step6 to step8, the maximum axial force increases $170 \mathrm{KN}$; From step8 to step10, the maximum axial force increases $40 \mathrm{KN}$; After step10, the axial force of the pile is basically stable. The increase of the axial force of the pile in the stage of the shield grouting and stripping is larger, which shows that the negative skin friction of foundation piles caused by shield tail construction cannot be neglected.

The bending moment distribution and variation law of shield passing through $1 \# \sim 10 \#$ pile as shown in Figure 8. It can be seen from the graph that the moment of the 1\#, 3\#, 5\#, 7\#and 9\# piles moment is almost equal to the bending moment of the $2 \#, 4 \#, 6 \#, 8 \#$ and $10 \#$ piles, indicating that the shielding effect in pile group foundation has limited effect on the moment. Comparing the net spacing between piles(3\#, 5\#, 7\# and 9\# pile) and tunnel, the net spacing increases gradually with the increase of stake number, and the variation degree of the corresponding pile bending moment decreases, indicating that the net spacing between the foundation pile and the tunnel is larger, the variation degree of the corresponding pile axial force is smaller.

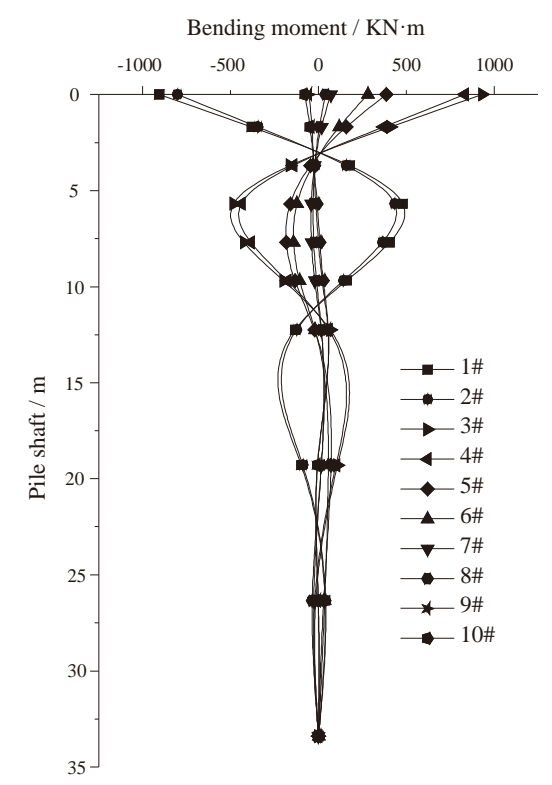

Fig. 8 Bending moment of each base pile

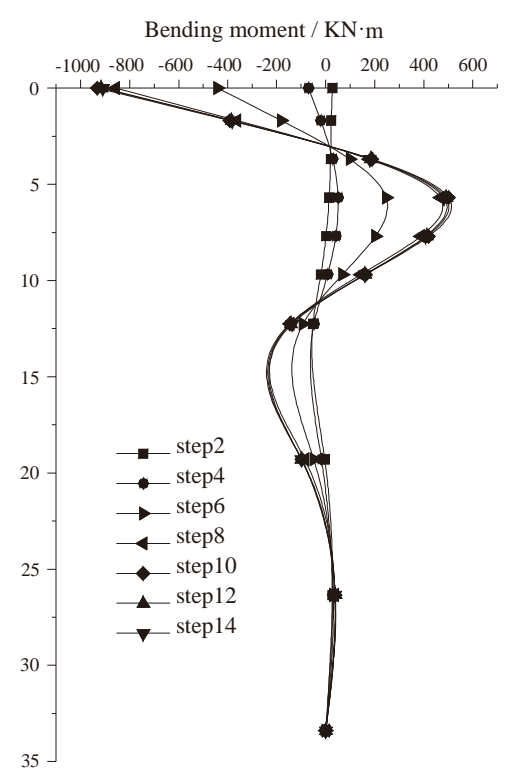

Fig. 9 Bending moment of $1 \#$ pile in the whole process of shield tunneling The curve of the bending moment of the whole process of the shield passing through the 1\# 
pile unit as shown in Figure 9. It can be seen from the figure that the bending moment of the pile is slightly changed at step2 and step 4; the bending moment increment is greater at step6; the bending moment of the pile is basically stable after step8. Therefore, the influence of shield tunneling on the moment of pile is mainly in the range of $6 \mathrm{~m}$ before and after the pile.

\section{Conclusion}

Combined with the actual project, numerical simulation is used to simulate and analyze the whole process of shield construction through the adjacent pile foundation. The main conclusions are as follows:

(1)Due to the restriction of the bridge group and the pile foundation to the soil, the nearer the soil is from the pile foundation, the smaller the ground subsidence is caused by the shield construction.

(2)There are shielding effect in group pile foundation, the shielding effect has great influence on the horizontal displacement and axial force of foundation pile, and the influence on the bending moment of each foundation pile can be neglected.

(3)with the increase of shield tunneling depth, the horizontal displacement of foundation pile gradually increases, then tends to be stable. In addition, the influence of shield tunneling on the horizontal displacement of pile mainly occurs within the range of $6 \mathrm{~m}$ before and after the pile.

(4)With the increase of the net spacing between the base pile and the tunnel, the axial force of each pile gradually decreases, and the change degree of the bending moment is gradually decreases.

\section{Reference documents:}

[1]Morton J D, King K H. Effects of tunneling on the bearing capacity and settlement of piled foundation[C]//I: Proceedings, Tunneling 79. London: IMM, 1979: 57-68.

[2]Loganathan N, Poulos H G, Stewart D P. Centrifuge model testing of tunnelling-induced ground and pile deformations[J]. Geotechnique, 2000, 50(3): 83-294.

[3]Ong C W, Leung C F, Yong K Y, Chow Y K. Centrifuge modeling of pile responses due to tunneling in clay[J]. Underground Singapore, 2005, 128(4): 327-336.

[4]Lee C J, Jacobsz S W. The influence of tunneling on adjacent piled foundations[J]. Tunneling and Underground Space Technology, 2006, 21: 430.

[5]Fang Yong, He Chuan. Study on the influence of Metro Shield tunnel construction on near-access Pile Foundation[J]. Modern tunnel technology, 2008, 45 (1): 42-47.

[6]Luo Wenlin, Liu Youwei, Han Xuan. Numerical analysis of influence of tunnel excavation on pile Foundation engineering[J]. Rock and soil mechanics, 2007, 28 (add): 403 -407.

[7]Zhao zhifeng. Numerical analysis of influence of Shield tunnel construction on Bridge Pile Foundation[J]. Journal of Wuhan University of Technology, 2010, (15): 47-50+59.

[8]Zhang Haibo, Liu Guonan, Gao Junhe. Analysis of influence of shield tunneling on bridge pile foundation[J]. Railway Engineering, 2007, (08): 37-40.

[9]Xu Ming, Xie Yongning. Influence of shield tunnel excavation on adjacent pile foundation[J]. Journal of South China University of Technology(Natural Science Edition), 2011, 39(4): 149-155. [10]Chen Yumin, Xu Dingping. FLAC/FLAC3D foundation and engineering example[M]. China Water Power Press, 2013. 11.

[11]Peck R B. Deep Excavations and Tunneling in Soft Ground[M]. New York: Published by ASCE, 1984. 